\title{
Identification of Single and Double Sites of Phosphorylation by ECD FT-ICR/MS in Peptides Related to the Phosphorylation Site Domain of the Myristoylated Alanine-Rich C Kinase Protein
}

\author{
Kellie A. Woodling, ${ }^{\mathrm{a}, *}$ John R. Eyler, ${ }^{\text {a }}$ Yury O. Tsybin, ${ }^{\mathrm{b},+}$ \\ Carol L. Nilsson, ${ }^{\mathrm{b}}$ Alan G. Marshall, ${ }^{\mathrm{c}}$ Arthur S. Edison, ${ }^{\mathrm{d}}$ \\ Iman M. Al-Naggar, ${ }^{e}$ and Michael R. Bubb \\ ${ }^{a}$ Department of Chemistry, University of Florida, Gainesville, Florida, USA \\ ${ }^{\mathrm{b}}$ National High Magnetic Field Laboratory, Tallahassee, Florida, USA \\ ${ }^{\mathrm{c}}$ National High Magnetic Field Laboratory and Department of Chemistry and Biochemistry, Florida State \\ University, Tallahassee, Florida, USA \\ ${ }^{\mathrm{d}}$ Department of Biochemistry and Molecular Biology, McKnight Brain Institute, and National High Magnetic \\ Field Laboratory, University of Florida, Gainesville, Florida, USA \\ ${ }^{\mathrm{e}}$ Department of Medicine, University of Florida, Gainesville, Florida, USA
}

\begin{abstract}
A series of phosphorylated test peptides was studied by electron capture dissociation Fourier transform ion cyclotron resonance mass spectrometry (ECD FT-ICR MS). The extensive ECD-induced fragmentation made identification of phosphorylation sites for these peptides straightforward. The site(s) of initial phosphorylation of a synthetic peptide with a sequence identical to that of the phosphorylation site domain (PSD) of the myristoylated alanine-rich C kinase (MARCKS) protein was then determined. Despite success in analyzing fragmentation of the smaller test peptides, a unique site on the PSD for the first step of phosphorylation could not be identified because the phosphorylation reaction produced a heterogeneous mixture of products. Some molecules were phosphorylated on the serine closest to the N-terminus, and others on one of the two serines closest to the C-terminus of the peptide. Although no definitive evidence for phosphorylation on either of the remaining two serines in the PSD was found, modification there could not be ruled out by the ECD fragmentation data. (J Am Soc Mass Spectrom 2007, 18, 2137-2145) (C 2007 American Society for Mass Spectrometry
\end{abstract}

$\mathrm{P}$ ost-translational modification of the MARCKS myristoylated alanine-rich $C$ kinase protein presents an interesting analytical challenge. The MARCKS protein is a well characterized acidic membrane protein comprised of 309 amino acids, and is believed to be natively unfolded [1]. It has been implicated in the regulation of brain development [2], cellular migration [3], cellular adhesion [4], tumor suppression [5], and neurosecretion [6]. This protein also binds calmodulin [7], acidic membrane phospholipids [8-10], and actin filaments [11]. These interactions are believed to be regulated by protein phosphorylation $[3,4,12$, 13]. The MARCKS protein is also a major substrate for protein kinase C (PKC) [14]. Protein kinase C-related kinase (PRK1) and $\rho$-associated kinase also phosphory-

Address reprint requests to Dr. J. R. Eyler, Department of Chemistry, University of Florida, P.O. Box 117200, Gainesville, FL 32611, USA. E-mail: eylerjr@chem.ufl.edu

* Current address: National Center for Toxicological Research, Jefferson, AR. + Current address: Biomolecular Mass Spectrometry Laboratory, Ecole Polytechnique Fédérale de Lausanne, CH-1015, Lausanne, Switzerland. late the phosphorylation site domain (PSD) $[15,16]$. PKC contains two regions: the N-terminal regulatory region and the $\mathrm{C}$-terminal catalytic region. This kinase phosphorylates serine and threonine residues in basic peptide and protein sequences. Thus, PKC phosphorylates a small segment of the MARCKS protein, displacing the protein from membranes. The phosphorylation of the MARCKS protein then inhibits cross-linking activity of filamentous (F) actin, calcium-calmodulin binding, and binding to the plasma membrane $[3,7$, 9, 11, 17-19].

The MARCKS protein contains three highly conserved regions: the phosphorylation site domain (PSD), the myristoylated amino-terminal domain, and the MARCKS homology domain. The PSD region, also known as the effector domain (ED), is the most studied, and is a basic peptide comprised of 25 amino acid residues. This region is believed to be central to the activity of the MARCKS protein. The binding of calmodulin and F-actin most likely occurs here [20, 21]. In addition, an electrostatic interaction between the posi- 
tively charged residues and the head groups of acidic phospholipids also occurs in this region [8-10]. The phosphorylation site domain contains five serine candidates for phosphorylation. Phosphorylation of this domain may be crucial to the overall function of the MARCKS protein [3, 4, 22, 23].

Previous studies have simply considered the function and behavior of the PSD region as an indicator of the overall function of the MARCKS protein. Although possibly the PSD peptide behaves differently than the intact protein, the analytical and biochemical methods developed for the smaller peptide may also be applicable for study of the intact MARCKS protein [12, 17, 24]. A previous mass spectrometric analysis suggested that the MARCKS protein may be phosphorylated at sites other than those of the PSD region. However, LC/ MS/MS spectra showed only $b$ and $y$-type ions. Phosphate loss was also seen, precluding localization of these modifications [1]. Prior fast-atom bombardment (FAB) mass spectrometry experiments indicated that serine residue 2 within the PSD sequence is phosphorylated first, followed by the phosphorylation of serine residue 1 [25].

Localization of post-translational modifications (PTMs) such as phosphorylation in protein and peptide molecules is often difficult and time-consuming by use of standard analytical techniques. Edman degradation and ${ }^{32} \mathrm{P}$ labeling are routinely used to locate phosphorylation sites [26]. Other techniques include the use of antibodies, sodium dodecylsulfate-polyacrylamide gel electrophoresis (SDS-PAGE), acid hydrolysis, X-ray crystallography, and NMR, all of which can require both lengthy analysis and large sample volumes [26-28]. Many of these problems can be overcome by mass spectrometric analysis [29].

Electrospray ionization (ESI) coupled to Fourier transform ion cyclotron resonance mass spectrometry (FT-ICR MS) has become a popular tool for analysis of many biomolecules, including peptides and proteins, due to its low extent of fragmentation, ease of remeasurement, high mass accuracy, and high mass resolving power [30-32]. Traditionally, collision-induced dissociation (CID) is used to sequence peptides and proteins $[29,33,34]$. Typically, CID heats the peptide to a higher Boltzmann temperature [35], often resulting in the loss of any labile modification during fragmentation. The presence of the modification is inferred by observing its loss from the parent ion, but knowledge of the site of the modification is lost. In contrast, low-energy electron capture dissociation (ECD) [36-38] achieves extensive peptide backbone fragmentation while leaving labile side-chain modifications intact, thereby enabling more complete sequencing of the peptides and localization of the modification. Typically, collisional dissociation generates $b$ - and $y$-type ions (i.e., cleavage of the C-N backbone bond) whereas ECD produces $c$ - and $z$-type ions (i.e., cleavage of the $\mathrm{C}-\mathrm{N}$ backbone bond) as well as $a$ (i.e., cleavage of the $\mathrm{C}-\mathrm{C}$ backbone bond) and $y$-type ions [33].
In this paper, we apply ECD to phosphorylated PSD with an amino acid sequence identical to that of the MARCKS protein, in an attempt to identify unique sites for the first and subsequent steps of phosphorylation. ECD of a series of singly and doubly phosphorylated test peptides with amino acid sequences similar to the PSD was followed by ECD of singly phosphorylated PSD. Phosphorylation sites in the singly and doubly modified test peptides were clearly identified, given the excellent ECD fragmentation efficiency we were able to obtain. However, results for the singly phosphorylated PSD segment were ambiguous, with evidence for several different sites of phosphorylation.

\section{Experimental}

\section{Sample Preparation}

Singly and doubly phosphorylated test peptides were modeled after the sequence of the nonphosphorylated PSD region (KKKKKRFSFKLSFKLSGFSFKKSKK) of the MARCKS protein. The MARCKS analogues (Table 1) each contained 13 amino acid residues, compared with the 25 amino acids present in the PSD sequence, and were synthesized to contain only three serine residues, with a maximum of two serine residues phosphorylated in any one peptide. The test peptides consisted of three singly and three doubly phosphorylated peptides, with a nonphosphorylated peptide used as a control. The amino acid sequences of all peptides were identical, differing only in the extent and location of phosphorylation. The MARCKS analogues were synthesized at the University of Florida Protein Core with phosphorylation at the desired serine residues achieved by incorporating phosphoserine residues into the peptide sequences during the synthesis.

The resulting phosphopeptides were purified by high-performance liquid chromatography (HPLC) with a $4.6 \mathrm{~mm}$ i.d. C-18 column and a flow rate of 1.0 $\mathrm{mL} / \mathrm{min}$. Gradient elution (10\%to $80 \%$ B) was utilized (Solvent A: $0.1 \%$ TFA $/ \mathrm{H}_{2} \mathrm{O}$, Solvent B: $0.1 \%$ TFA/ $\mathrm{ACN}$ ), with a total elution period of $32 \mathrm{~min}$.

MALDI-TOF analysis served to confirm the molecular mass of each purified peptide. The MALDI-TOF analyses were performed with a Voyager ED-PRO MALDI-TOF instrument (Applied Biosystems, Foster

Table 1. Predicted monoisotopic masses for the seven MARCKS analogues. The phosphoserine residues are denoted by pS

\begin{tabular}{clc}
\hline $\begin{array}{c}\text { MARCKS } \\
\text { analogue }\end{array}$ & \multicolumn{1}{c}{ Sequence } & $\begin{array}{c}\text { Monoisotopic } \\
\text { mass (Da) }\end{array}$ \\
\hline \hline 1 & KKSFKLSGFSFKK & 1530.8922 \\
2 & KKpSFKLSGFSFKK & 1610.8248 \\
3 & KKSFKLpSGFSFKK & 1610.8248 \\
4 & KKSFKLSGFpSFKK & 1610.8248 \\
5 & KKpSFKLpSGFSFKK & 1690.8585 \\
6 & KKpSFKLSGFpSFKK & 1690.8585 \\
7 & KKSFKLpSGFpSFKK & 1690.8585 \\
\hline
\end{tabular}


City, CA) operating in reflector mode with delayed extraction. An accelerating voltage of $20,000 \mathrm{~V}$ was applied to the ions from each MALDI spot ( $\alpha$-cyano-4hydroxycinnamic acid matrix), with an extraction period of 200 ns. Mass spectra were co-added from 200 laser shots per acquired spectrum.

\section{PSD Phosphorylation}

An oligopeptide with an amino acid sequence identical to that of the phosphorylation site domain (PSD) region of the MARCKS protein was synthesized at the University of Florida Protein Core. Purification of the synthetic PSD region again utilized HPLC. MALDI-TOF analysis was used to confirm the molecular mass of the peptide. The synthetic PSD segment was diluted to a concentration of $1 \mathrm{mg} / \mathrm{mL}$ in $5 \mathrm{mM}$ MES (2-morpholinoethane sulfonic acid) buffer ( $\mathrm{pH}$ 6.0) for use in the phosphorylation reaction. The phosphorylation reaction was carried out in $50 \mathrm{mM}$ MES (pH 6.0), $1.25 \mathrm{mM}$ EGTA (ethylene glycol-bis ( $\beta$-aminoethylether)-N, $\mathrm{N}, \mathrm{N}^{\prime}, \mathrm{N}^{\prime}$ tetraacetic acid) buffer ( $\mathrm{pH} 7.5), 12.5 \mathrm{mM} \mathrm{MgCl}, 0.125$ $\mathrm{mM}$ ATP with $30 \mathrm{ng}$ of rat brain catalytic subunit of PKC (Calbiochem, San Diego, CA). The reaction was incubated for $4 \mathrm{~h}$ at $30^{\circ} \mathrm{C}$ in an agitating water bath. The reaction was quenched by boiling at $100^{\circ} \mathrm{C}$ for 15 min and centrifuging at $14,000 \mathrm{rpm}$ for $15 \mathrm{~min}$ in a tabletop microcentrifuge. The supernatants were collected and desalted before mass spectrometric analysis.

\section{Electron Capture Dissociation Fourier Transform Mass Spectrometry}

Electron capture dissociation experiments were carried out with a custom-built 9.4 T ESI-Q-FTICR mass spectrometer at the National High Magnetic Field Laboratory (NHMFL) $[39,40]$. Final peptide concentration for electrospray ionization (Nanomate robot from Advion Biosciences, Ithaca, NY) was 5 to $10 \mu \mathrm{M}$ in 1:1 acetonitrile:water with the addition of $0.1 \%$ formic acid. The ECD experiments were performed with a $10 \mathrm{~mm}$ diameter dispenser cathode (Heat Wave Labs, Watsonville, CA) mounted on the rear of the analyzer cell. The desired charge state was isolated by an external massselective quadrupole mass filter [41]. The cathode was operated at a potential of $-5 \mathrm{~V}$ during the electron capture events ( $20 \mathrm{~ms}$ irradiation period). A total of 100 single scan acquisitions were co-added for each tandem mass spectrum.

\section{Data Analysis}

Data analysis was carried out with modular ICR data acquisition system (MIDAS) data analysis software developed at the NHMFL [42, 43]. Each spectrum was baseline corrected, Hanning apodized [44] and zerofilled twice. The thorough high resolution analysis of spectra by Horn (THRASH) program was used to generate the experimental peak lists obtained for the ECD experiments [45]. The monoisotopic masses of fragment ions were imported into the ProSight PTM program for generation of the fragmentation summaries [46]. Mass tolerance was 0.1 Da for identifying the fragment ions. The ProSight program was used to identify the $c / z$ and $b / y$ ion pairs whereas the $a$-type ions were identified from the Protein Prospector database [47].

\section{Results and Discussion}

Figure 1 shows a tandem mass spectrum of a nonphosphorylated reference peptide. Nine $c$-type and $10 z$-type ions were observed, providing complete sequencing. A series of seven $a$-type ions was also observed. The resulting fragmentation summary, showing all observed fragment ions, can be seen in the inset of Figure 1.

\section{Fragmentation of Singly Phosphorylated Polypeptides}

Figure 2 shows the ECD mass spectrum and resulting fragmentation summary for the $[\mathrm{M}+3 \mathrm{H}]^{3+}$ charge state of MARCKS analogue 2 (sequence shown in Table 1). Cleavages occurred at all backbone bonds, resulting in the formation of $10 c$-type and ten $z$-type ions.

In addition to the most frequent ECD fragment ions, nine $a$-type and six $y$-type ions were also seen. The fragment ions resulting from cleavage on both the $\mathrm{N}$-terminal and C-terminal sides of the modified residue correctly indicate the presence of the modification on Ser 3. There are no fragments consistent with phosphorylation of either Ser 7 or Ser 10. Similar scenarios were seen for MARCKS analogues 3 and 4 . In each case, the site of phosphorylation was correctly assigned to Ser 7 or Ser 10, respectively.

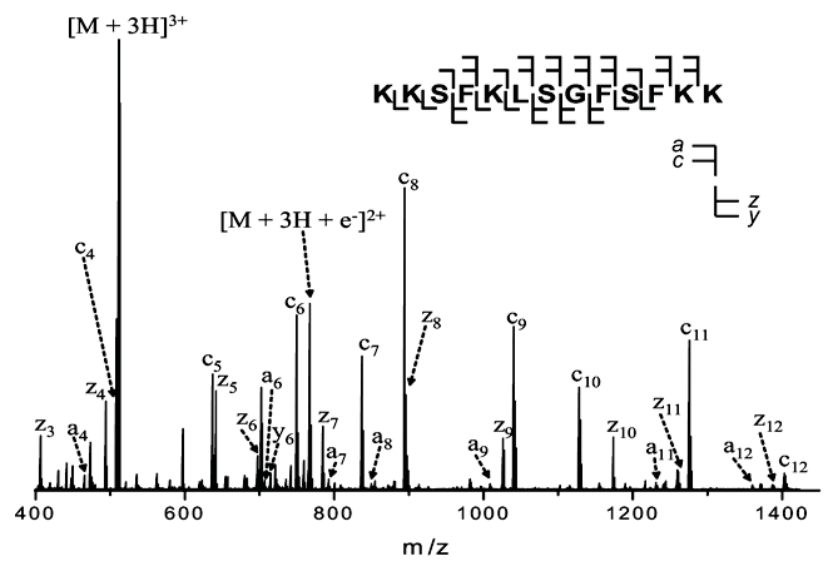

Figure 1. ECD FT-ICR mass spectrum of MARCKS analogue 1. All N-C $\alpha$ backbone bonds were cleaved. All labeled fragment ions are singly charged. As shown in the inset, a single bracket denotes that only c or z ions were seen for that bond cleavage. The double bracket denotes the presence of $a$ - or $y$-ions in addition to $c$ - or $z$-ions. The same fragmentation designation is used in Figures 2, 3, and Schemes 1-4. 


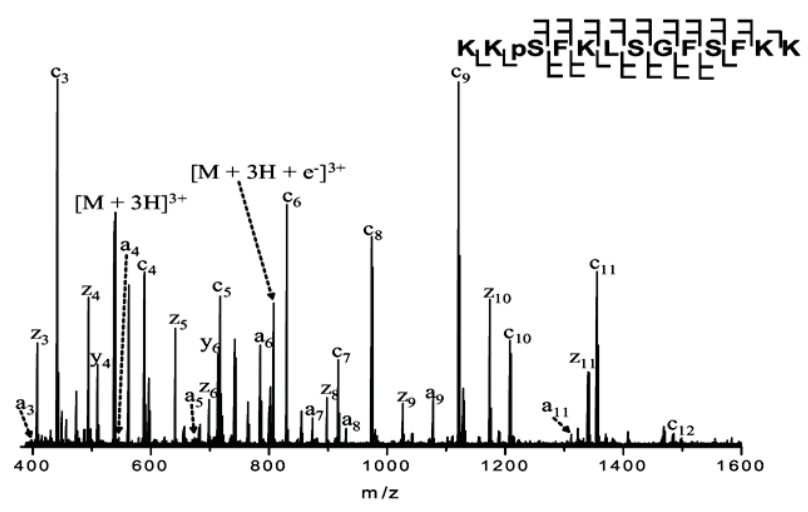

Figure 2. ECD FT-ICR mass spectrum of MARCKS analogue 2. All N-C $\alpha$ backbone bonds were cleaved, allowing localization of the site of phosphorylation to Ser 3. All fragment ions are singly charged. The phosphoserine residue (Ser 3) is denoted by pS.

\section{Fragmentation of Doubly Phosphorylated Polypeptides}

Figure 3 shows the ECD mass spectrum for MARCKS analogue 5 (sequence shown in Table 1). Ser 3 and Ser 7 were phosphorylated in this peptide, whereas Ser 10 was not modified. Cleavage occurred at all backbone bonds, allowing for complete sequencing of this phosphopeptide.

A total of 34 fragment ions was formed, permitting localization of the sites of phosphorylation. The fragment ions on the $\mathrm{N}$-terminal $\left(z_{11}\right)$ and C-terminal $\left(c_{3}\right)$ sides of Ser 3 indicate the presence of a phosphate group. Fragment ions $z_{7}$ and $c_{7}$ indicate the presence of a modification on Ser 7. The fragment ions surrounding Ser 10 show an unmodified residue at that position. Similar scenarios were seen for MARCKS analogues 6 and 7 (sequences shown in Table 1). In each case, the sites of phosphorylation could be localized to the correct serine residues (Scheme $\mathbf{1}$ ).

The relative abundances of the resulting fragment ions were explored in an attempt to determine if there

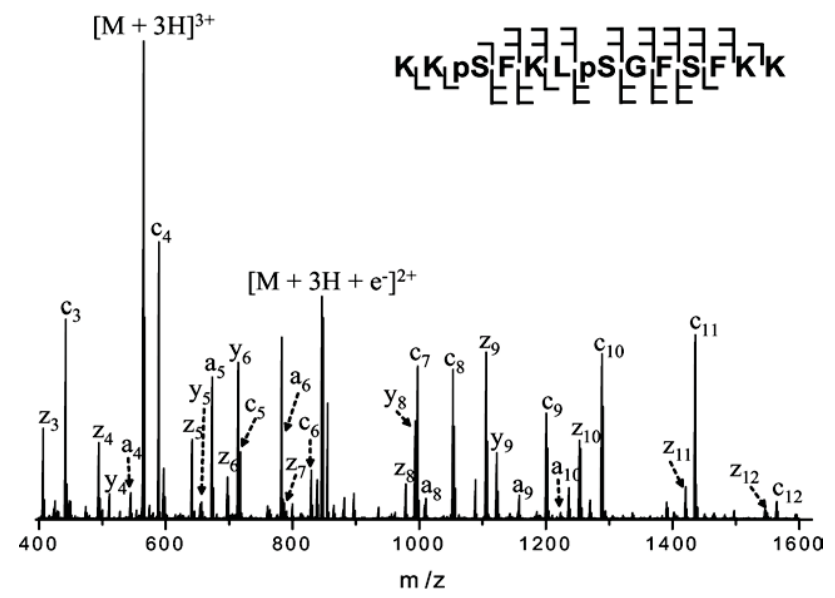

Figure 3. ECD FT-ICR mass spectrum of MARCKS analogue 5. The sites of phosphorylation were correctly assigned to Ser 3 and Ser 7. All fragment ions are singly charged.
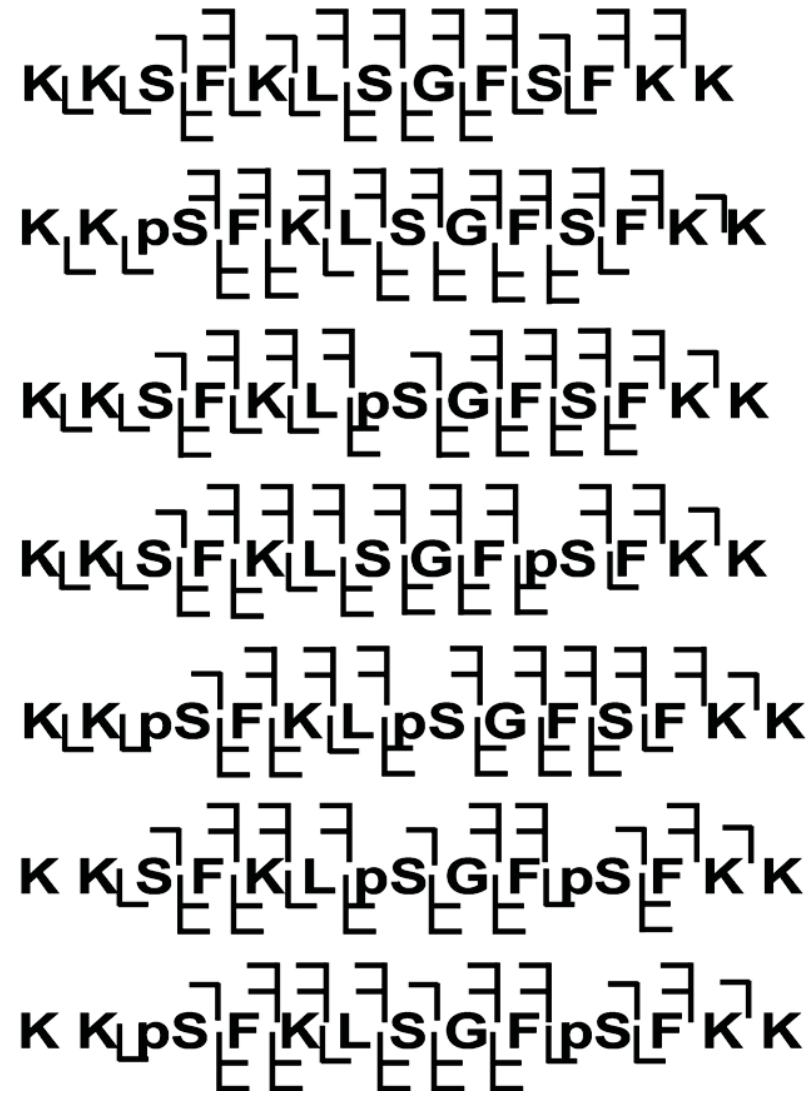

Scheme 1. Fragmentation summaries obtained for the electron capture dissociation of the MARCKS analogues. In each case, the sites of phosphorylation could be localized to the correct serine residues.

was a distinct pattern to the retention or loss of the phosphate group. The relative abundances for the $a$ type ions of the doubly phosphorylated MARCKS analogue peptides (Figure 4) provide information regarding the abundances of the modified residues.

The only a-type ions were from the unmodified residues. There were no $a$-ions representing fragmentation on the C-terminal side of the phosphorylated residues in each of the doubly phosphorylated peptides. As an example, for MARCKS analogue 7, fragment ions $a_{3}$ and $a_{7}$ would represent fragmentation

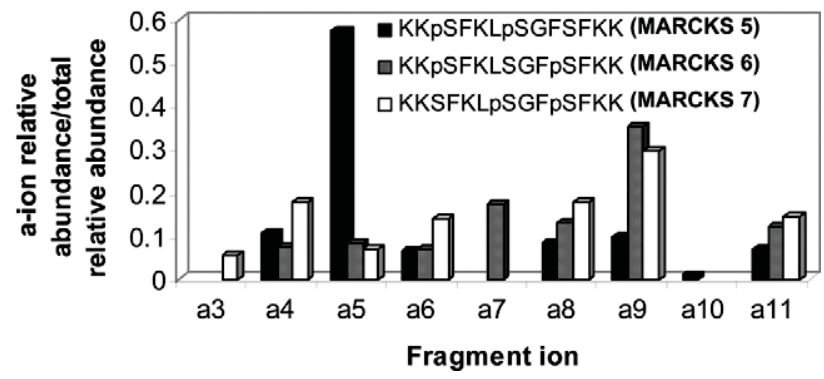

Figure 4. $a$-Ion relative abundance histogram for the doubly phosphorylated MARCKS analogues. The $a$-ions provide complementary structural information for the phosphorylation site determination. 
following Ser 3 and Ser 7. In each case, the $a$-type ion is not present. However, there is a fragment ion in the experimental peak list that corresponds to the loss of the modification from the serine residue ( $a_{7}$-phosphate). It could not be determined if the same is true for fragment ion $a_{3}$, because that $m / z$ value falls below the scanned mass range.

It should be noted that a similar trend was not seen for the singly phosphorylated peptides. Their $a$-type ions corresponded to cleavage on the C-terminal side of the modified residues. There was no evidence that the phosphate groups were lost during the dissociation process, as for the doubly phosphorylated peptides. The trend for the doubly phosphorylated peptides may or may not hold true for other modified peptides. Because the loss of the modification is usually not seen in electron capture dissociation, future work should focus on determining the relative abundances of the $a$-type ions for other multiply-modified peptides.

\section{Differentiation of Polypeptides with Identical Mass and Number of Modifications}

The results reported above clearly indicate (as have earlier studies) [33, 48-51] that ECD can be used to confirm known structures of phosphorylated peptides. However, analyzing peptides with unknown sites of phosphorylation can nevertheless occasionally be problematic. As seen in Table 1, MARCKS analogues 2, 3, and 4 each have the same molecular mass and number of modifications as do MARCKS analogues 5, 6, and 7. We simulated the analysis of peptides with unknown sites of phosphorylation, based on our test peptides, by comparing the predicted ECD fragments for two of the mono- (or di-)phosphorylated peptides with the actual experimental ECD fragments obtained from the third mono- (or di-)phosphorylated peptide. All possible comparisons were made.

The experimental peak lists obtained for each of the singly phosphorylated peptides were compared with those predicted for each of the other two singly modified peptides in turn to determine how many of the experimental fragment ions were identical to those predicted for each peptide. Based upon the theoretically obtained peak lists, several fragment ions for each of the singly phosphorylated peptides were predicted to be identical, whereas the remaining ions differ (due to different sites of phosphorylation) for each of the respective peptides. The fragment ions unique to each peptide should have been found only in the experimentally generated peak list for the "correct" peptide. As an example, fragment ions $c_{7}$ and $z_{11}$ should be identical for peptides 2 and 3 whereas fragment ion $c_{3}$ should be different. The fragment ions that differ from peptide to peptide should provide a means of unambiguously differentiating between each of the similar peptides. An analogous scenario should hold true for the doubly phosphorylated peptides.

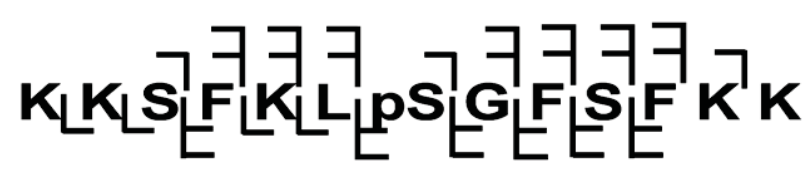

MARCKS analogue 3
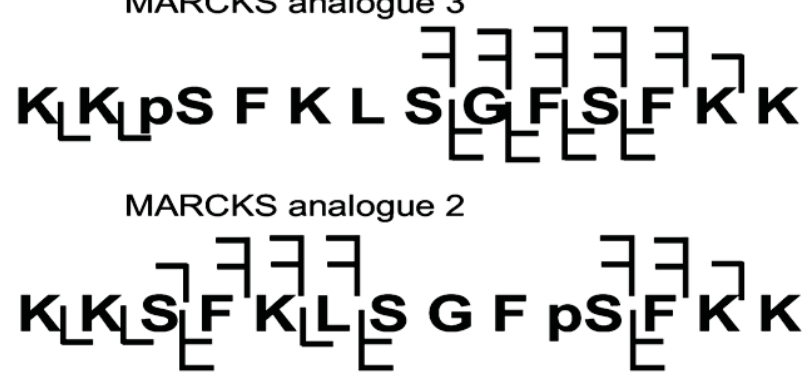

MARCKS analogue 4

Scheme 2. ECD correlation analysis for MARCKS analogues $2-4$. The peak list obtained from the ECD of MARCKS analogue 3 was compared with that predicted for analogues 2 and 4 . The fragment ions shown are those that match the experimental peak list for analogue 3.

The experimental peak list for MARCKS analogue 3 was compared with those predicted for peptides 2 and 4 (Scheme 2) to determine if the site of phosphorylation could be unambiguously determined. For peptide 2, 21 predicted fragment ions matched those obtained for peptide 3. Each of the 21 fragment ions could be attributed to peptide 3 .

The presence of fragment ion $z_{11}$ indicates that any of the three serine residues may be phosphorylated whereas fragment ion $c_{7}$ indicates that the lone modification may be located on either Ser 3 or Ser 7 . The exact location cannot be determined based upon the theoretically generated list of fragment ions. A similar scenario was seen for peptide 4 (with the peak list for peptide 3). Twenty-two fragment ions matched, with no discrepancies. Fragment ion $z_{7}$ indicates phosphorylation on Ser 7 or Ser 10 whereas fragment ion $c_{10}$ indicates that any of the three serine residues may be modified. Further localization is not possible (Scheme 2).

An analogous procedure was carried out for each of the doubly phosphorylated peptides. For example, the experimentally obtained peak list for MARCKS analogue 6 was compared with those theoretically predicted for MARCKS analogues 5 and 7. Scheme 3 shows the predicted fragments for each of the doubly phosphorylated peptides that match the experimentally obtained peak list for peptide 6 . For the predicted peaks from peptide 5 (based on the experimental peak list for peptide 6), 22 fragment ions matched. All fragment ions produced by electron capture dissociation were consistent with fragmentation from MARCKS analogue 6 , but there was no agreement between experimental peaks from analogue 6 and predicted peaks from analogue 5 that would suggest phosphorylation on the second serine in the sequence (appropriate to analogue 5) rather than the third serine (appropriate to analogue 6 , and from which the experimental peak 


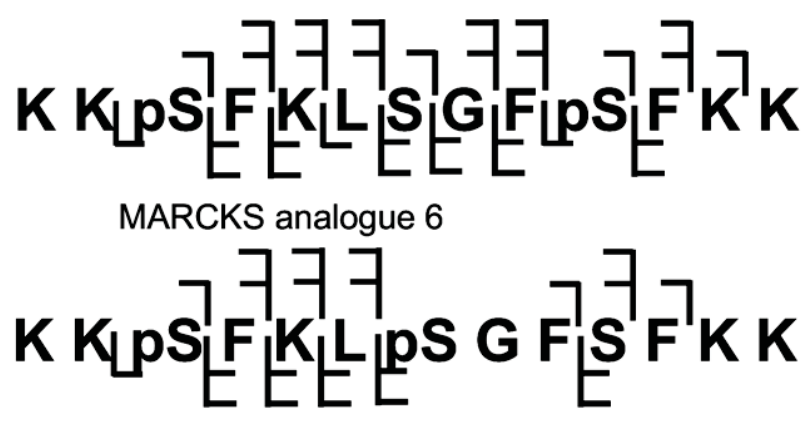

MARCKS analogue 5

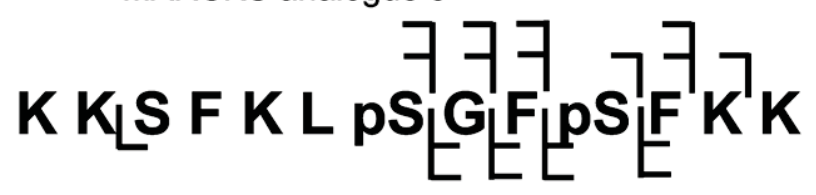

MARCKS analogue 7

Scheme 3. ECD correlation analysis for MARCKS analogues 5-7. The peak list obtained for the ECD of analogue 6 was compared with those predicted for analogues 5 and 7 . The fragment ions shown are those that match the experimental peak list for analogue 6 .

list was taken). Thus incorrect sites of phosphorylation could not be assigned if the "wrong" predicted peak list were compared with the experiment data. If the predicted fragment ions for peptide 7 were compared with the experimental peak list for peptide 6 , 19 fragment ions matched, but again no fragments were seen that would lead to mis-assignment of the sites of phosphorylation.

Schemes $\mathbf{2}$ and $\mathbf{3}$ illustrate the need for fragment ions on both sides of the modified amino acid residues for complete localization of the modification (or modifications). For each scenario, the "missing" fragment ions would be present in the predicted peak lists of the "wrong" peptides only if the sites of phosphorylation were different from those in the peptide for which experimental data were obtained. Although a few discrepancies did arise (data not shown), the sites of phosphorylation for the actual sequences, based on the experimentally obtained peak lists, were correctly identified only by use of the "correct" peak lists.

Given the success of identifying the sites of phosphorylation in singly and doubly modified MARCKS PSD analogues, and the ability to tell that singly and doubly phosphorylated sites of "unknowns" were not at possible locations of a peptide with known phosphorylation sites, we next proceeded to analysis of the singly phosphorylated peptide with a sequence identical to the PSD region of the MARCKS protein.

\section{Application of Fragmentation Guidelines to the PSD Polypeptide}

The synthetic PSD segment was phosphorylated in vitro and subjected to electron capture dissociation mass spectrometry. The extent of phosphorylation was not known before the mass spectrometric analysis. The precursor mass spectrum (data not shown) showed both a singly phosphorylated and a doubly phosphorylated PSD segment. The abundances of doubly phosphorylated ions were insufficient for reliable ECD fragmentation. The 4+ charge state of the singly phosphorylated PSD segment was isolated by SWIFT excitation $[52,53]$ and subjected to ECD, Figure 5.

Because the PSD region contains five serines that may be phosphorylated, all resulting in ions with identical mass, five different peptide sequences are possible for this polypeptide. In an approach similar to that described above for the test peptides, we compared the experimentally obtained fragment peak list in turn with that predicted for PSD phosphorylated at each of the five serine residues.

The experimental fragment ions matching those predicted for serine 1 phosphorylation (and a similar representation for the PSD phosphorylated at each of the other four serine residues) are shown in Scheme 4. The presence of $c_{8}(\mathrm{~m} / \mathrm{z} 1128.7008), c_{9}, c_{10}$, and $c_{11}$ fragment ions indicates that some portion of the sample mixture is phosphorylated at Ser 8. There are fragment ions that correspond to cleavage on either the $\mathrm{N}$ terminal or C-terminal sides, or both, of the remaining (unmodified) serine residues.

Fragment ion $c_{12}$ might suggest modification at Ser 12. However, that ion mass is identical to that of the $c_{12}$ fragment that would be obtained from PSD phosphorylated at Ser 8 (as are the $c_{13}$ through $c_{24}$ fragment ions), so the presence of those ions does not prove that an additional fraction of the sample is phosphorylated at Ser 12. However, some of the peptide molecules are phosphorylated at a serine residue other than Ser 8, as evidenced by the presence of $c_{8}(m / z 1048.7478), c_{9}, c_{10}$,

\section{p[KKKKKRFSFKKSFK L S GFSFKKSK K]}

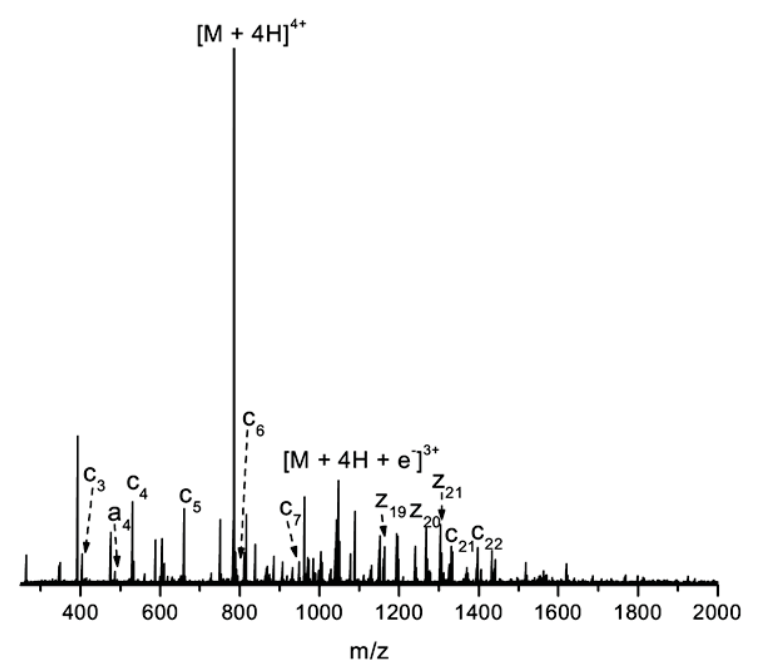

Figure 5. ECD mass spectrum for the singly phosphorylated PSD segment. The precursor ion was isolated by SWIFT excitation before electron capture dissociation. 


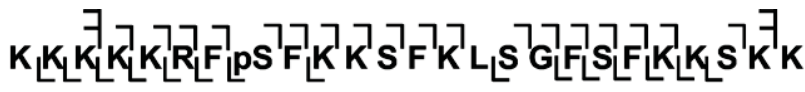

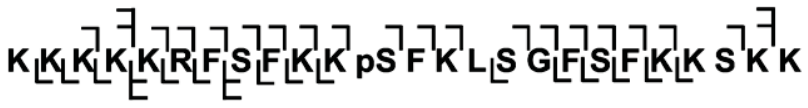

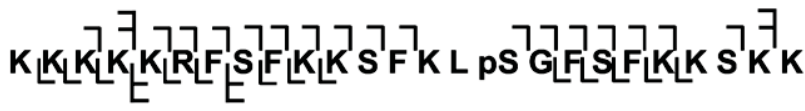

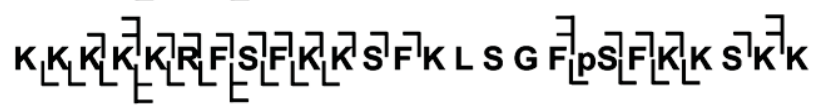

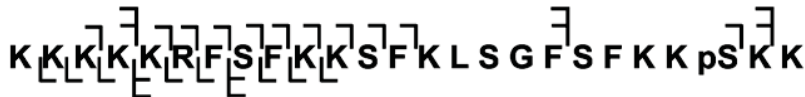

Scheme 4. Fragmentation summaries for each unique sequence combination for the singly phosphorylated PSD segment. Each fragmentation summary was produced by comparing the experimentally obtained peak list from the electron capture dissociation experiment with the theoretically predicted peak list for each sequence combination.

and $c_{11}$ ions, all with masses consistent with no phosphorylation at Ser 8. In addition, it should be noted that the presence of fragment ions $z_{15}, z_{16}$, and $z_{17}$ indicates that one of Ser 12, Ser 16, Ser 19, or Ser 23 is modified. The mixture is apparently heterogeneous, making assignment of the fragment ions to one particular PSD sequence impossible. No fragment ions corresponding to two cleavages, one on either side of a phosphorylated "internal" serine residue, were seen. Thus it is not possible to assign single phosphorylation unambiguously to Ser 12, Ser 16, or Ser 19.

Because positive identification of serine phosphorylation at Ser 8 was possible from comparison of experimental fragment ions with those predicted for a single cleavage for that species $\left(c_{8}, c_{9}, c_{10}\right.$, and $c_{11}$ ions, as discussed above), the species predicted for ECD of ions phosphorylated at the other "external" serine residue, Ser 23, are next compared with those found experimentally (Scheme 4). The absence of experimental fragment ions with masses corresponding to those predicted for $z_{3}, z_{4}, z_{5}$, or $z_{6}$ ions from PSD modified at Ser 23 strongly suggests that none of the singly phosphorylated PSD peptide molecules are modified at Ser 23. Possibly a very small fraction of the sample is phosphorylated at Ser 23, but the ECD fragment ions produced from it do not have sufficient signal-to-noise to be detected. That ions with masses identical to those predicted for $c_{18}$ (and $a_{18}$ ) fragments are seen in the experimental spectrum indicates that some portion of the sample is phosphorylated at either Ser 19 or Ser 23. Similar observation of ions with masses identical to those predicted for $c_{12}$ and $c_{13}$ indicates that some fraction of the sample is phosphorylated at either Ser 16, Ser 19, or Ser 23.

The fragmentation summaries for Ser 16 and Ser 19 phosphorylation (also shown in Scheme 4) provide no additional information; each is consistent with possible phosphorylation at any of the five sites. However, the agreement of the predicted $z_{7}$ fragment ion peak for phosphorylation at Ser 19 with one observed experimentally reinforces the conclusion drawn in the last paragraph that some fraction of the sample is phosphorylated on either Ser 19 or Ser 23.

It is apparent from the results presented above that in vitro phosphorylation of the PSD peptide results in single phosphorylation of at least two different serine residues. At least some of the phosphorylation takes place at Ser 8, and a second portion of the sample is phosphorylated at either Ser 19 or Ser 23, with Ser 19 most likely since no direct evidence was seen for phosphorylation at Ser 23. Although no definitive evidence was found for phosphorylation of Ser 12 or Ser 16 , those modifications cannot be ruled out based on the ECD peaks seen.

These results show that while ECD fragmentation patterns may be quite unambiguous for small peptides with one or two known sites of phosphorylation, definitive assignment of phosphorylation sites for larger peptides with numerous possible sites for modification is far from straightforward. Future experiments will attempt to obtain doubly phosphorylated PSD peptides in sufficient quantity to provide additional information about the second site of phosphorylation and will explore more vigorous ECD conditions that might produce fragmentation of two bonds on either side of phosphorylated "internal" serine residues. It is also possible that detailed examination of the abundances of ECD fragment ions might provide additional information as to the probability of phosphorylation at each possible site, similar to results recently reported for histone acetylation [54].

Our results are not in agreement with the previous FAB MS study [25] that predicted that Ser 12 was the first to be phosphorylated in the PSD peptide. Although we cannot rule out the possibility that some fraction of the sample is initially phosphorylated on Ser 12 , it is also clear that other fractions are initially phosphorylated on Ser 8 and on Ser 19 or Ser 23 (probably Ser 19). The previous study was based on a PSD segment that contained 21 amino acid residues, rather than the 25 amino acid peptide in the current set of experiments. In addition, the peptide studied previously contained four serine residues (consistent with human PSD), not five like ours (consistent with murine PSD), that could potentially be phosphorylated. Those differences in the PSD segments could affect the phosphorylation site(s) found by mass spectrometric analysis. However, it is unlikely that the discrepancies can be explained by major structural differences between the peptides because they are both likely to exhibit highly extended structures in solution.

\section{Conclusions}

Electron capture dissociation Fourier transform ion cyclotron resonance mass spectrometry was successfully utilized to determine the sites of phosphorylation 
for a series of singly and doubly phosphorylated test peptides (MARCKS analogues), based on extremely efficient fragmentation. In all cases both the singly and doubly modified polypeptide fragment ions on both the $\mathrm{N}$-terminal and C-terminal sides of the modified residue (or residues) could be used to identify the site(s) of phosphorylation unambiguously.

ECD experiments based on the same approach were applied to a singly phosphorylated peptide with a sequence identical to that of the phosphorylation site domain (PSD) of the MARCKS protein, containing five potentially modifiable serine residues. The efficiency of fragmentation was reduced compared with the smaller test peptides, and we were unable to obtain a peak of sufficient magnitude for the doubly phosphorylated PSD peptide to enable ECD experiments. We found for the singly phosphorylated peptide that phosphorylation was not localized exclusively on any one site. The results indicate that some of the peptide molecules are modified at Ser 8 and others at either Ser 19 or Ser 23, most likely Ser 19. Phosphorylation at Ser 12 or Ser 16 cannot be ruled out, although no direct evidence for it was seen. The addition of the phosphate group is apparently not directed to one thermodynamically or kinetically favored serine during the in vitro phosphorylation reaction. Although the heterogeneity of the sample mixture complicated interpretation of the ECD data, it was still possible to gain some knowledge as to the exact site (or sites) for the initial step of phosphorylation of this peptide.

\section{Acknowledgments}

The authors thank Alfred Chung for the synthesis of the MARCKS analogues and Stanley Stevens for the use of the MALDI-TOF mass spectrometer. This work was supported in part by the NSF National High-Field FT-ICR Mass Spectrometry Facility (DMR 00-84,173), Florida State University, and the National High Magnetic Field Laboratory at Tallahassee, Florida.

\section{References}

1. Matsurbara, M.; Yamauchi, E.; Hayashi, N.; Taniguchi, H. MARCKS, a Major Protein Kinase C Substrate, Assumes Nonhelical Conformations Both in Solution and in Complex with $\mathrm{Ca}^{2+}$-Calmodulin. FEBS Lett. 1998, 421, 203-207.

2. Stumpo, D. J.; Bock, C. B.; Tuttle, J. S.; Blackshear, P. J. MARCKS Deficiency in Mice Leads to Abnormal Brain Development and Perinatal Death. Proc. Natl. Acad. Sci. U.S.A. 1995, 92, 944-948.

3. Arbuzova, A.; Schmitz, A. A.; Vergeres, G. Cross-talk Unfolded: MARCKS Proteins. Biochem. J. 2002, 362, 1-12.

4. Arbuzova, A.; Murray, D.; McLaughlin, S. MARCKS, Membranes and Calmodulin: Kinetics of Their Interaction. Biochim. Biophys. Acta 1998, 1376, 369-379.

5. Brooks, G. The Role of $80 \mathrm{~K} / \mathrm{MARCKS}$, a Specific Substrate of Protein Kinase C, in Cell Growth and Tumor Progression. Pigment Cell Res. 1994, 7, 451-457.

6. Trifaro, J. M.; Lejen, T.; Rose, S. D.; Pene, T. D.; Barkar, N. D.; Seward, P. Pathways that Control Cortical F-actin Dynamics During Secretion. Neurochem. Res. 2002, 27, 1371-1385.

7. Graff, J. M.; Young, T. N.; Johnson, J. D.; Blackshear, P. J. PhosphorylationRegulated Calmodulin Binding to a Prominent Cellular Substrate for Protein Kinase C. J. Biol. Chem. 1989, 264, 21818-21823.

8. Taniguchi, H.; Manenti, S. Interaction of Myristoylated Alanine-Rich Protein Kinase C Substrate (MARCKS) with Membrane Phospholipids. J. Biol. Chem. 1993, 268, 9960-9963.

9. Kim, J.; Shishido, T.; Jiang, X.; Aderem, A.; McLaughlin, S. Phosphorylation, High Ionic Strength, and Calmodulin Reverse the Binding of
MARCKS to Phospholipid Vesicles. J. Biol. Chem. 1994, 269, 28214-28219.

10. Arbuzova, A.; Wang, L.; Wang, J.; Hangyas-Mihalyne, G.; Murray, D. Honig, B.; McLaughlin, S. Membrane Binding of Peptides Containing Both Basic and Aromatic Residues. Experimental Studies with Peptides Corresponding to the Scaffolding Region of Caveolin and the Effector Region of MARCKS. Biochemistry 2000, 39, 10330-10339.

11. Hartwig, J. H.; Thelen, M.; Rosen, A.; Janmey, P. A.; Nairn, A. C.; Aderen, A. MARCKS is an Actin Filament Crosslinking Protein Regulated by Protein Kinase C and Calcium-calmodulin. Nature 1992, 356, $618-622$.

12. Tapp, H.; Al-Naggar, I. M.; Yarmola, E. G.; Harrison, A.; Shaw, G. Edison, A. S.; Bubb, M. MARCKS is a Natively Unfolded Protein with an Inaccessible Actin-binding Site. J. Biol. Chem. 2005, 280, 9946-9956.

13. Arbuzova, A.; Wang, J.; Murray, D.; Jacob, J.; Cafiso, D. S; McLaughlin, S. Kinetics of Interaction of the Myristoylated Alanine-rich C Kinase Substrate, Membranes and Calmodulin. J. Biol. Chem. 1997, 43, $271677-$ 227177.

14. McIlroy, B. K.; Walters, J. D.; Blackshear, P. J.; Johnson, J. D. Phosphorylation-dependent Binding of a Synthetic MARCKS Peptide to Calmodulin. J. Biol. Chem. 1991, 266, 4959-4964.

15. Palmer, R. H.; Schonwaber, D. C.; Rahman, D.; Pappin, D. J. C.; Herget, T.; Parker, P. J. PRK1 Phosphorylates MARCKS at the PKC Sites: Serine 152, Serine 156 and Serine 163. FEBS Letters 1996, 378, 281-285.

16. Nagumo, H.; Ikenoya, M.; Sakurada, K.; Furuya, K.; Ikuhara, T.; Hirgaka, H.; Sasaki, Y. Rho-associated Kinase Phosphorylates MARCKS in Human Neuronal Cells. Biochem. Biophys. Res. Commun. 2001, 280, 605-609.

17. Taniguchi, H.; Manenti, S.; Suzuki, M.; Titani, K. Myristoylated Alanine-rich C Kinase Substrate (MARCKS), a Major Protein Kinase C Substrate, is an in vivo Substrate of Proline-directed Protein Kinases: A Mass Spectrometric Analysis of Post-translational Modifications. J. Biol. Chem. 1994, 269, 18299-18302.

18. Newton, A. C. Protein Kinase C: Structure, Function, and Regulation J. Biol. Chem. 1995, 270, 28495-28498.

19. Rosen, A.; Keenan, K. F.; Thelen, M.; Nairn, A. C.; Aderem, A Activation of Protein Kinase C Results in the Displacement of its Myristoylated, Alanine-Rich Substrate from Punctate Structures in Macrophage Filopodia. J. Exp. Med. 1990, 172, 1211-1215.

20. Qin, K.; Cafiso, D. S; Qin, Z. Membrane Structure of the Protein Kinase $C$ and Calmodulin Binding Domain of Myristoylated Alanine-Rich C Kinase Substrate Determined by Site-directed Spin Labeling. Biochemistry 1996, 35, 2917-2925.

21. Yarmola, E. G.; Edison, A. S.; Lenox, R. H.; Bubb, M. R. Actin Filament Cross-Linking by MARCKS: Characterization of Two Actin-Binding Sites Within the Phosphorylation Site Domain. J. Biol. Chem. 2001, 276, 22351-22358.

22. Bubb, M. R.; Lenox, R. H.; Edison, A. S. Phosphorylation-Dependent Conformational Changes Induce a Switch in the Actin-binding Function of MARCKS. J. Biol. Chem. 1999, 274, 36472-36478.

23. Seki, K.; Sheu, F.; Huang, K. Binding of Myristoylated Alanine-Rich Protein Kinase C Substrate to Phosphoinositides Attenuates the Phosphorylation by Protein Kinase C. Arch. Biochem. Biophys. 1996, 326 , 193-201.

24. Yamauchi, E.; Kiyonami, R.; Kanai, M.; Taniguchi, H. The C-terminal Conserved Domain of MARCKS is Phosphorylated in Vivo by ProlineDirected Protein Kinase. J. Biol. Chem. 1998, 273, 4367-4371.

25. Arness, B.; Manjarrez-Hernandex, A.; Howell, S. A.; Learmonth, M.; Aitken, A. Multisite Phosphorylation of the $80 \mathrm{kDa}$ (MARCKS) Protein Kinase C Substrate in C3H/10T1/2 Fibroblasts. FEBS Lett. 1992, 297, 285-291.

26. Shi, S. D. H.; Hemling, M. E.; Carr, S. A.; Horn, D. M.; Lindh, I.; McLafferty, F. W. Phosphopeptide/phosphoprotein Mapping by Electron Capture Dissociation Mass Spectrometry. Anal. Chem. 2001, 73, 19-22.

27. Mann, M.; Jensen, O. N. Proteomic Analysis of Post-Translational Modifications. Nature Biotech. 2003, 21, 255-261.

28. McLachlin, D. T.; Chait, B. T. Analysis of Phosphorylated Proteins and Peptides by Mass Spectrometry. Curr. Opin. Chem. Biol. 2001, 5, 591-602.

29. Siuzdak, G. The Expanding Role of Mass Spectrometry in Biotechnology; MCC Press: San Diego, 2003.

30. Marshall, A. G.; Hendrickson, C. L.; Jackson, G. S. Fourier Transform Ion Cyclotron Resonance Mass Spectrometry: A Primer. Mass Spectrom. Rev. 1998, 17, 1-35.

31. Marshall, A. G.; Hendrickson, C. L. Fourier Transform Ion Cyclotron Resonance Detection: Principles and Experimental Configurations. Int J. Mass Spectrom. 2002, 215, 59-75.

32. Amster, I. J. Fourier Transform Mass Spectrometry. J. Mass Spectrom 1996, 31, 1325-1337.

33. Cooper, H. J.; Hakansson, K.; Marshall, A. G. The Role of Electron Capture Dissociation in Biomolecular Analysis. Mass Spectrom. Rev. 2005, 24, 201-222

34. Laskin, J.; Futrell, J. H. Collisional Activation of Peptide Ions in FTICR Mass Spectrometry. Mass Spectrom. Rev. 2003, 22, 158-181.

35. Goeringer, D.; Duckworth, D.; McLuckey, S. Collison-Induced Dissociation in Quadrupole Ion Traps: Application of a Thermal Model to Diatomic Ions. J. Phys. Chem. A 2001, 105, 1882-1889.

36. Zubarev, R. A.; Kelleher, N. L.; McLafferty, F. W. Electron Capture Dissociation of Multiply Charged Protein Cations. A Nonergodic Process. J. Am. Chem. Soc. 1998, 120, 3265-3266. 
37. Tsybin, Y. O.; Ramstron, M.; Witt, M.; Baykut, G.; Hakansson, P. Peptide and Protein Characterization by High-rate Electron Capture Dissociation Fourier Transform Ion Cyclotron Resonance Mass Spectrometry. J. Mass Spectrom. 2004, 39, 719-729.

38. McLafferty, F. W.; Horn, D. M.; Breuker, K.; Ge, Y.; Lewis, M. A.; Cerda, B.; Zubarev, R. A.; Carpenter, B. K. Electron Capture Dissociation of Gaseous Multiply Charged Ions by Fourier Transform Ion Cyclotron Resonance. J. Am. Soc. Mass Spectrom. 2001, 12, 245-249.

39. Senko, M. W.; Hendrickson, C. L.; Pasa-Tolic, L.; Marto, J. A.; White, F. M.; Guan, S.; Marshall, A. G. Electrospray Ionization Fourier Transform Ion Cyclotron Resonance Mass Spectrometry at 9.4 Tesla. Rapid Commun. Mass Spectrom. 1996, 10, 1824-1828.

40. Hakansson, K.; Chalmers, M. J.; Quinn, J. P.; McFarland, M. A.; Hendrickson, C. L.; Marshall, A. G. Combined Electron Capture and Infrared Multiphoton Dissociation for Multistage MS/MS in a Fourier Transform Ion Cyclotron Resonance Mass Spectrometer. Anal. Chem. 2003, 75, 3256-3262.

41. Senko, M. W.; Hendrickson, C. L.; Emmett, M. R.; Shi, S. D.-H.; Marshall, A. G. External Accumulation of Ions for Enhanced Electrospray Ionization Fourier Transform Ion Cyclotron Resonance Mass Spectrometry. J. Am. Soc. Mass Spectrom. 1997, 8, 970-976.

42. Senko, M. W.: Canterbury, J. D.; Guan, S.; Marshall, A. G. A High Performance Modular Data System for Fourier Transform Ion Cyclotron Resonance Mass Spectrometry. Rapid Commun. Mass Spectrom. 1996, 10, 1839-1844.

43. Blakney, G. T.; van der Rest, G.; Johnson, J. R.; Freitas, M. A.; Drader, J. J: Shi, S. D. H.; Hendrickson, C. L.; Kelleher, N. L.; Marshall, A. G. Further Improvements to the MIDAS Data Station for FT-ICR Mass Spectrometry. Proceedings of the 49th American Society for Mass Spectrometry Conference on Mass Spectrometry and Allied Topics; Chicago, IL, June 2001.

44. Burton, R. D.; Matuszak, K. P.; Watson, C. H.; Eyler, J. R. Exact Mass Measurements Using a 7 Tesla Fourier Transform Ion Cyclotron Reso- nance Mass Spectrometer in a Good Laboratory Practices Regulated Environment. J. Am. Soc. Mass Spectrom. 1999, 10, 1291-1297.

45. Horn, D. M.; Zubarev, R. A.; McLafferty, F. W. Automated Reduction and Interpretation of High Resolution Electrospray Mass Spectra of Large Molecules. J. Am. Soc. Mass Spectrom. 2000, 11, 320-332.

46. LeDuc, R. D.; Taylor, G. K.; Kim, Y.; Januszyk, T. E.; Bynum, L. H.; Sola, J. V.; Garavelli, J. S.; Kelleher, N. L. ProSight PTM: An Integrated Environment for Protein Identification and Characterization by Top Down Mass Spectrometry. Nucleic Acids Res. 2004, 32, W340-W345.

47. Clauser, K. R.; Baker, P. R.; Burlingame, A. L. Role of Accurate Mass Measurement $(+/-10 \mathrm{ppm})$ in Protein Identification Strategies Employing MS or MS/MS and Database Searching. Anal. Chem. 1999, 71, 2871-2882.

48. Stensballe, A. Jensen, O. N.; Olsen, J. V ; Haselmann, K. F.; Zubarev, R. A. Electron Capture Dissociation of Singly and Multiply Phosphorylated Peptides. Rapid Commun. Mass Spectrom. 2000, 14, 1793-1800.

49. Kelleher, N. L. Top Down Proteomics. Anal. Chem. 2004, 76, 197A-203A.

50. Horn, D. M.; Lindh, I.; McLafferty, F. W. Phosphopeptide/Phosphoprotein Mapping by Electron Capture Dissociation Mass Spectrometry. Anal. Chem. 2001, 73, 19-22.

51. Chalmers, M. J.; Kolch, W.; Emmett, M. R.; Marshall, A. G.; Mischak, H Identification and Analysis of Phosphopeptides. J. Chromatogr. B 2004, 803, 111-120.

52. Guan, S.; Marshall, A. G. Stored Waveform Inverse Fourier Transform (SWIFT) Ion Excitation in Trapped Ion Mass Spectrometry Theory and Applications. Int. J. Mass Spectrom. Ion Processes 1996, 158, 5-37.

53. Marshall, A. G.; Wang, T.-C. L.; Ricca, T. L. Tailored Excitation for Fourier Transform Ion Cyclotron Resonance Mass Spectrometry. J. Am. Chem. Soc. 1985, 107, 7893-7897.

54. Pesavento, J. J.; Mizzen, C. A.; Kelleher, N. L. Quantitative Analysis of Modified Proteins and Their Positional Isomers by Tandem Mass Spectrometry: Human Histone H4. Anal. Chem. 2007, 78, 4271-4280. 